Aus der chirurgischen Universitätsklinik Frankfurt a. M. Direktor: Geh. Medizinalrat Prof. Dr. L. Rehn.

\title{
Zur Ätiologie und Diagnose des Zenkerschen Pulsionsdivertikels des Ösophagus.
}

\author{
Von Dr. Hans Kloiber, Assistenzarzt der Klinik.
}

(Mit 2 Abbildungen.)

Das Ösophagusdivertikel zählt zu den nicht alltäglichen Erkrankungen. Nach der Behauptung Zenkers ist das Pulsionsdivertikel der Speiseröhre so selten, daß die meisten Ärzte überhaupt keines zu Gesicht bekommen. Dieser Satz besaß wohl für früher seine Gültigkeit, hat aber in letzter Zeit entschieden daran eingebüßt. Während nämlich $\mathrm{Starck}$ im Jahre 1900 93 Divertikel aus der Literatur zusammenstellte, konnte Lotheissen im Jahre 1913 deren bereits 210 vorfinden und seither haben die Publikationen weiterhin zugenommen. Diese Zunahme darf aber nicht darin gesucht werden, daß das Ösophagusdivertikel nun wirklich häufiger auftritt wie früher, sondern hat ihre einfache Erklärung und Ursache in der Verbesserung und dem Ausbau unserer Diagnostik. Und hier gebührt das. größte Verdienst neben der Ösophagoskopie in erster linie der Radiographie. Von beiden Methoden hat es gerade die letztere vielfach erst ermöglicht, eine klare Diagnose zu stellen, wo man früher auf Vermutungen angewiesen war.

In folgendem will ich über 2 Fälle von Pulsionsdivertikel des Ösophagus berichten, bei deren Untersuchung das Röntgenverfahren sehr wichtige Dienste geleistet und Aufschlüsse gegeben hat, die wir mit den sonst üblichen Untersuchungsmethoden nicht erhalten können.

Im I. Fall handelt es sich um einen 82 jährigen Mann, der am 26. XI. 19I6 wegen eines Abszesses an der großen Zehe des linken. Fußes auf die Chirurg. Univ.-Klinik zur Aufnahme kommt. 
Unabhängig von diesem Leiden klagt er aber über Beschwerden beim Schlucken und die deswegen eingehend erhobene Anamnese ergibt folgendes:

Der Patient ist früher stets gesund gewesen. Seit 5 Jahren leidet er an Schluckbeschwerden. Anfangs konnte er feste Speisen noch hinunter bringen, in letzter Zeit jedoch nicht mehr. Jetzt machen ihm auch schon breiige Speisen Beschwerden. Er hat das Gefühl, als ob die Speisen im Halse stecken blieben und beim Essen hat er immer einen Druck im Halse. I ic Speisen kommen nach einiger Zeit wieder unverdaut hoch und er muß sie 2-3 mal kauen, bis er sie cndlich hinunter bringt. Daher braucht er zum Essen eine beträchtliche Zeit und mul sich dabei meist recht anstrengen. Die hochgekommenen Speisen schmecken weder sauer noch bitter und weisen reinen Speisegeschmack auf. Flüssigkeiten gehen am besten hinunter, aber auch bei diesen merkt er ein Steckenbleiben im Halse. Beim Essen und Trinken muß er den Kopf weit nach vorne halten, weil dann die Speisen und Getränke am leichtesten nach unten gehen. Früher hat er immer schr rasch gegessen und sehr große Bissen geschluckt. Schon seil langer Zeit hat er überhaupt keine Zähne mehr. Bis vor 2 Jahren war sein Ernährungszustand recht gut. Seit dem Kriege ist aber sein Körpergewicht beträchtlich zurückgegangen.

B of un d: Der Kranke ist ein großer, mittelkräftiger Mann von stark reduziertem Ernährungszustand. Haut und sichtbare Schlcimhäute sind sehr blaß3. Dic Pupillen sind gleich weit und reagieren auf I.ichteinfall prompt. Beide Gesichtshälften fühlen sich gleich warm an. Der Mund ist vollkommen zahnlos. Die Zunge ist weißlich belegt, jedoch feucht. Ziemlich starker Foetor ex ore. Der Hals ist nicht besonders dick. Die Schilddrüsenlappen sind in normaler Größe palpabel. Xeben der Schilddrüse läßt sich keine Geschwulst fühlen.

Keinc abnorme Dämpfung nachweisbar. Läßt man den Patienten ein Glas Wasser trinken, so setzt er sich im Bette auf und beugt den Kopf und die Brust ganz nach vorn, weil er dann das Wasser besser hinunter bringt. Trinkt ar in gewöhnlicher aufrechter Stellung oder gar im Liegen, so hat er nach dem Trinken sofort das Gefühl, als ob das Wasser im Halse stecken bliebe. Nach kurzer Zeit kommen Teile desselben mit etwas Schleim vermengt wieder hoch. Als Stelle, wo das Wasser stecken bleibt, bezeichnet er die Gegend des Kehlkopfes. Beim Schlucken hört man an dieser Stelle kein Cicräusch. Auch beim Auskultieren läßt sich kein Blasen und Quatschen feststellen. Gibt man feste Speisen zu essen, so hat er viel eher das Gefühl des Steckenbleibens und diese kommen so, wie sie gegessen wurden, wieder zum Vorschein. In ausgespuckten Schleim befinden sich viele Spcisereste. Läßt man endlich breige Speisen nehmen und perkutiert nun, so findet man eine leichte Dämpfung auf ter linken Halsseite, die sich beim Schlucken etwas verschiebt. Fine Gischwulst 
ist aber auch jetzt nicht wahrzunehmen. Es besteht leichte Heiserkeit. Die Untersuchung der ausgespuckten Speiseteile mit Lackmuspapier ergibt neutrale Reaktion. Beim Einführen der Magensonde stößt man $22 \mathrm{~cm}$ von der Zahnreihe entfernt auf einen elastischen, federnden Widerstand.

Röntgenuntersuchung: Bci der Durchleuchtung sieht man nach Aufnahme einer Wismutaufschwemmung deutlich, daß ein Teil der Flüssigkeit die Speiseröhre glatt passiert. Ein größerer Teil aber weicht nach hinten $a b$ und bildet einen birnenförmigen

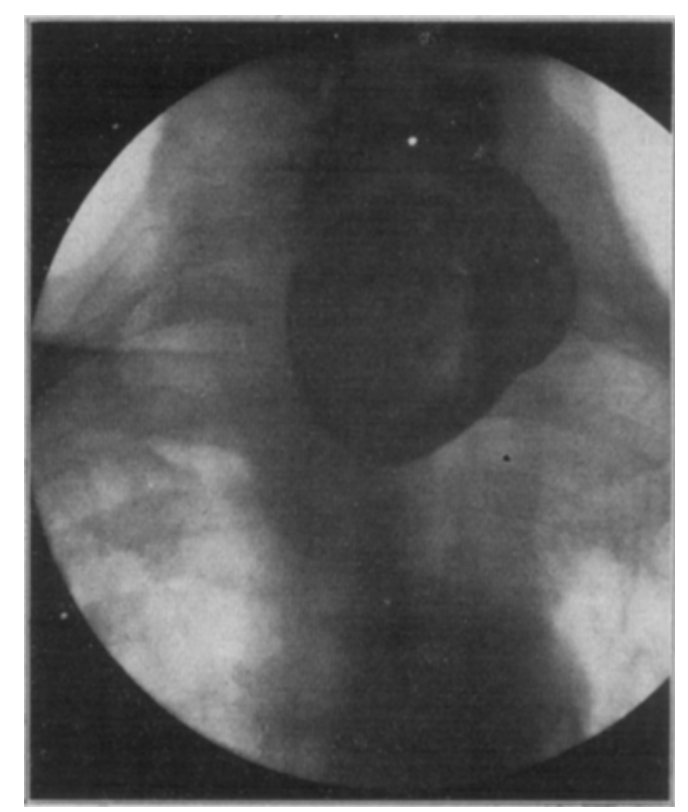

Fig. 1.

Schatten, dessen nach unten halbmondförmige Begrenzung vollkommen glatt ist, während die obere eine unregelmäBige Linie darstellt. Eine Luftblase über dem Schatten ist nicht zu erkennen. Der Schatten, der bis zur oberen Thoraxapcrtur herabreicht, steigt beim Schlucken gleichmäßig auf und ab. Dabei bemerkt man zeitweise, daß der Sack sich nach oben entleert und sozusagen wie eine volle Schale überläuft. Bei der Durchleuchtung in Fechterstellung sieht man nach dem Scblucken einer Wismutaufschwemmung einen Schatten vor dem birnenförmigen Gebilde heruntergleiten. Das Röntgenogramm zeigt bei ventrodorsaler Aufnahme im Stehen einen ziemlich großen halbmondförmigen Schatten, der vom Processus transversus des 7 . Hals. 
wirbels bis zur Höhe der oberen Thoraxapertur herabreicht. Die untere Begrenzungslinie ist vollkommen scharf, die obere dagegen ist unscharf gezeichnet und bildet keine reine Horizontale, sondern ist an den Seiten leicht sichelförmig ausgebogen. Nach links reicht der Schatten um ungefähr ein Drittel weiter als nach rechts.

Zwischen unterer Schattengrenze und dem Aortenbogen liegt

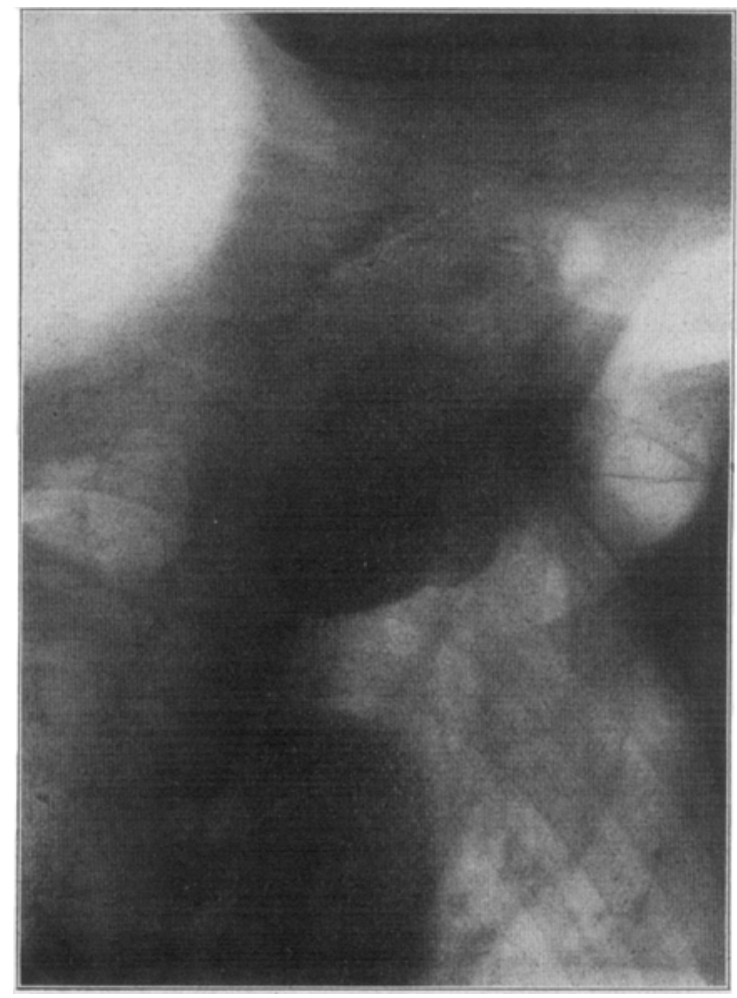

Fig. 2.

ein ungefähr zweiquerfingerbreiter, heller Raum. Auf einer 2. Röntgenaufnahme findet man den unteren Kontur doppelt umrissen. Der Patient hat bei der Aufnahme geschluckt und es ist auf diese Weise ein Diplogramm entstanden, das in deutlicher Weise die Steighöhe des Schattens fixiert. Die untere Grenze ist nämlich dabei um ungefähr $3 \mathrm{~cm}$ nach oben gestiegen. Ein weiteres Röntgenbild (Fig. I), das im Liegen aufgenommen wurde, zeigt einen gänseeigroßen, herzförmigen Schatten. Die Umrisse sind sehr scharf von der Umgebung abgehoben, während das Innere wolkiges Aussehen aufweist. Die 
obcre Hälfte dieses Schattens ist kleiner wie die untere und beide sind voneinander durch eine zwerchsackähnliche Einschnürung in der Mitte deutlich abgegrenzt. Von der Mitte der oberen Hälfte geht ein kleiner Zapfen nach oben und verbreitert sich dann zu einem größeren Band, das sowohl nach rechts wie nach links mit nach außen leicht konvexer Linie scharf umschrieben ist, während es nach oben nur eine horizontale unklare Linie zeigt. Bei seitlicher Aufnahme im Liegen (Fig. 2) sieht man am Röntgenbild ebenfalls wieder einen herzförmigen, in der Mitte zwerchsackähnlichen eingeschnürten Schatten, dessen obere Hälfte nun größer ist als die untere. Die Umrisse sind wicder recht scharf ausgeprägt, das Innere, wie oben beschrieben, wolkig gezeichnet. Der Abstand zwischen Aortenbogen und unterer Grenze des Schatten beträgt 2 Querfingerbreite.

Nach diesem Befunde muß also ein Pulsationsdivertikel des Ösophagus vorliegen.

Bei der Röntgenuntersuchung am nächsten 'Tage ist das Divertikel noch zur Hälfte gefüllt. Nach 5 Tagen findet man im Divertikel noch einen kleinen schalenförmigen Rest, ähnlich dem Rückstand des Kontrastbreies bei Gastrektasie. Dieser Schatten liegt direkt hinter dem oberen Rande des Brustbeins. Erst am 6. Tage läßt sich im Divertikel kein Schatten mehr nachweisen.

Der Kranke lehnt von vornherein jeden operativen Eingriff ab, bevor man ihm überhaupt noch einen diesbezüglichen Vorschlag gemacht hat.

Aus dem weiteren Verlaufe interessiert, daß wegen fortschreitender Abszedierung noch 3 gröBere Incisionen am Fuße gemacht werden müssen. Es kommt zu Gangrän des Fußes, die allmählich weiter fortschreitet. Der Patient wird immer elender, dic Körperkräfte verfallen ziemlich rasch und am I8.XII. I916 kommt es zum Exitus letalis.

Kurze Zeit nach dieser Beobachtung bekam ich nach dem Gesetz der Duplizität der Fälle ein zweites Ösophagusdivertikel zu Gesicht.

Ein 72 jähriger Metzger, der früher nie besonders krank war, klagt seit 4 Jahren über Schluckbeschwerden. Er kann wenigèr gut schlucken wie früher. Feste Speisen und Brot wollen nicht recht hinuntergehen, ohne Flüssigkeit schon gar nicht. Wenn er mittags gegessen hat, bekommt er öfters Hustenreiz, als wenn cr sich verschluckt hätte. Für gewöhnlich kommen keine Speisen herauf. Dann und wann jedoch verspürt er nach dem Essen einen Kitzel im Halse, bekommt dann Husten und muß sich übergeben. Das Erbrochene enthält die frisch gegessenen Speisen. Dies passiert ihm jedoch nur bei festen Speisen. Beim Essen von Suppe und Trinken von Flüssigkeit ,,kommt nichts vor." In letzter Zeit muß er auch im Bette öfters räuspern und 
es kommen dann Speisen hoch. Die Speisen riechen nicht unangenehm und sind mit wenig Schleim vermengt. Beim Schlucken von fester Nahrung hat er das Gefühl, als ob sie im Halse stecken blieben. Geräusche hört er nicht beim Schlucken. Er klagt über viel SpeichelfluB. Sein Körpergewicht ist nicht zurückgegangen. Weiter gibt er an, schon seit langen Jahren ein schlechtes Gebiß zu haben. Er hat immer sehr rasch gegessen und große Bissen geschluckt. Er ist der Ansicht, daß seine Beschwerden vom raschen Essen herkommen.

B e f und: Patient ist ein mittelgroßer, kräftig gebauter Mann von mittlerem Ernährungszustand.

Haut und sichtbare Schleimhäute sind gut durchblutet. prompt.

Die Pupillen sind gleich weit und reagieren auf Lichteinfall

Das Gebiß ist äußerst mangelhaft.

Die Zunge ist feucht und nicht belegt. Es besteht nur geringer Foetor ex ore. Am Halse ist eine Geschwulst weder zụ sehen noch zu palpieren. Auch nach dem Schlucken von Wasser, sowie nach dem Essen von Brei tritt am Halse keine Vorwölbung und keine Dämpfung auf. Eine Druckempfindlichkeit ist nirgends vorhanden. Die Schilddrüsenlappen zeigen normale Größe. Beim. Schlucken hört man auf der Höhe des Kehlkopfes ein deutliches, glucksendes Geräusch. Lungen und Herz zeigen keine krankhaften Veränderungen. Nervenstatus ohne Besonderheiten. Bei der Sondenuntersuchung stößt man $21 \mathrm{~cm}$ hinter der Zahnreihe auf einen Widerstand. Auch bei einer 2. Sondierung findet man diesen Widerstand. Bei der Sondenuntersuchung am nächsten Tage fühlt man kein Hindernis und die Sonde gleitet glatt in den Magen.

Da Verdacht auf ein Ösophagusdivertikel besteht, wird cine Röntgenuntersuchung der Speiseröhre vorgenommen. Nach Schlucken ciner Wismutaufschwemmung sieht man vor dem Schirm bei der dorso-ventralen Durchleuchtung an der oberen Thoraxapertur einen ungefähr taubeneigroßen Schatten, dessen unterer Rand mit nach unten konvexer Linie scharf begrenzt ist, und dessen oberer Rand horizontal verläuft. Über diesem Schatten befindet sich eine deutliche Luftblase. Beim Schluckakt steigt der Schatten deutlich auf- und abwärts. Bei der Durchleuchtung in Fechterstellung zcigt sich deutlich, wie beim Schlucken von Wismutaufschwemmung ein Schattenband vor diesen taubeneiförmigen Schatten heruntergleitet. Diagnose: Pulsionsdivertikel des Ösophagus.

Der Kranke kann sich zur Operation nicht entschließen, weil er doch verhältnismäßig wenige Schmerzen habe. Sollten stärkere Beschwcrden auftreten, dann will er zur Operation in die Klinik kommen. M. erscheint am 25. VI. I917 zur Nachuntersuchung und gibt an, die Beschwerden seien die gleichen wie früher. Die Röntgenuntersuchung ergibt kein Wachstum des Divertikels. 
In beiden Fällen liegt also ein Ösophagusdivertikel vor, und zwar das typische Zenkersche Grenzdivertikel. Grenzdivertikel wird es genannt, weil es an der Grenze von Pharynx und Speiseröhre sich findet. Auf diese seine Lage ist atuch sein anderer Name, ,pharyngo-ösophageales Divertikel“ zurückzuführen. Diese Divertikel gehören in die Kategorie der Pulsionsdivertikel. Sie stellen eine Ausstülpung der Hinterwand des Ösophagus dar; ihre Wand besteht aus Schleimhaut, aber es sind larin auch Muskelfasern gefunden worden.

Was dic Ätiologie dieser Erkrankung betrifft, so sind vera schiedene Erklärungsversuche gemacht worden, auf die ich nicht näher eingehen will. Sta $\mathrm{ck}$ hat dieselben in seiner eingehenden Monographie genau beschrieben und das Für und Wider besprochen. Ich möchte nur kurz Stellung nehmen zu der einen Theorie, die ziemlich viele Anhänger hat, nämlich daß die Ur^ sache für diese Divertikel in einer embryonalen Anlage und Entwicklungsstörung zu suchen sei. Daß eine kongenitale Anlage in einigen Fällen vorliegt, mag ohne weiteres zugegeben werden, und die eine Beobachtung Berg man $\mathrm{n}$ s liefert dafür auch einen Beweis. Ich möclite aber doch recht bezweifeln, daß dies die Regel ist. Zieht man nämlich eine kongenitale Anlage zur Erklärung heran, so ist nicht einzusehen, warum sich nicht viel früher an dieser Stelle ein Ösophagusdivertikel entwickelt, sondern sich erst im vorgeschrittenen Alter einstellt.

Bei dieser Annahme liegt aber auch ferner gar kein Grund vor, warum die Männer bedeutend häufiger an Ösophagusdivertikel erkranken wie die Irauen. Entfallen doch von ten 79 Pulsionsdivertikel, bei denen Starck in den Publikationen das Geschlecht festgestellt fand, 60 auf Männer und nur I 9 auf Frauen. Andere Untersucher kommen zu den gleichen Ergebnissen, und auch in meinen Fällen handelt es sich beide Male um Männer. Es müssen also noch unbedingt andere Schädigungen mitwirken und diese Schädigungen sind sicherlich mechanischer Natur. Hierher gehört meiner Ansicht nach schnelles Essen. Und gerade Männer sind recht häufig ausgesprochene Schnell. esser, weil der Zeitmangel in ihrem Berufe sie dazu verführt. Vor allem aber spielt auch das in höherem Alter meist vorhandene schlechte Gebiß eine Rolle. Was diesen Punkt betrifft, so macht 
St a r ck noch sehr spärliche diesbezügliche Angaben, wohl aus dem Grunde, weil man früher den Zähnen für die Entstehung von Krankheiten noch nicht diese große Bedeutung zulegte wie heute. Spätere Untersucher betonen dies aber immer mehr und mehr. So findet man in den meisten neueren Veröffentlichungen immer wieder die Bemerkung in der Anamnese, da $B$ die Kranken ein schlechtes Gebiß hatten und daß die Speisen mit großer Hast eingenommen wurden.

Killian konnte fast regelmäßig bei den von ihm beobachteten Ösophagusdivertikeln ein schlechtes Gebiß feststellen und in der Krankengeschichte die Bemerkung verzeichnen, daß die Patienten immer recht hastig gegessen und große Bissen geschluckt haben. Mit Recht mißt er daher diesem Umstand große Bedeutung bei.

$\mathrm{Hu}$ be $\mathrm{r}$ behandelte einen Patienten mit Ösophagusdivertikel, der ein ganz ungewöhnlicher Schnellesser war und seiner Ansicht nach hat dies bei der Entstehung des Divertikels wesentlich mitgewirkt. Auch Payr, Maruyama, Albrecht und andere glauben, daß in der Ätiologie des Pulsionsdivertilsels, schlechtes GebiB und schnelles Essen eine Rolle spielen.

Auch meine beiden Patienten geben klar und eindeutig an, daß sie seit langem ein schlechtes Gebiß besäßen und daß sie immer recht rasch gegessen und große Bissen geschluckt hätten. Der eine hatte überhaupt keine Zähne mehr und der andere nur noch einige wenige, die kein normales Kauen mehr erlaubten.

Diesem Umstand muß für die Entstehung der Ösophagusdivertikel sicherlich mehr Gewicht beigelegt werden als bisher geschehen. Ich neige der Anschauung $z u$, daß überhaupt mechanische Momente in erster Linie bei der Bildung eines Divertikels verantwortlich zu machen sind, wie das bereits 2 enker und $\mathrm{Starck}$ getan haben.

Die Zenkerschen Grenzdivertikel finden sich stets auf der Höhe des Ringknorpels. Diese Stelle entspricht genau dem Übergang des Pharynx in die Speiseröhre und liegt unmittelbar über der ersten physiologischen Stenose des Ösophagus. Der Entstehungsmechanismus ist wohl folgender:

Bei schlechtem oder mangelhaftem Gebiß können die Speisen 
nicht ordentlich gekaut werden und es werden daher ziemlicl große Bissen geschluckt. Werden nun die Bissen allzu groß, so ist sehr leicht die Möglichkeit gegeben, daß sie sich an der ersten physiologischen Enge der Speiseröhre fangen, wie wir ja öfters ein Steckenbleiben von Speisen, hauptsächlich an der dritten physiologischen Enge, knapp oberhalb der Cardia beobachten. Bleiben die Speisen an der Stenose liegen, so wird durch weiteres Schlucken ein gewisser Überdruck hergestellt, der zu einer Dehnung der Speiseröhre führt. Da der Ösophagus nicht überall gleiche Wandstärke besitzt, wird sich auch dieser Druck nicht gleichmäßig verteilen und zu einer diffusen Dilatation führen, sonclern er wird sich am chesten und am stärksten an der schwächsten Sielle bemerkbar machen. Und grerade über der ersten Enge der Speiseröhre findet sich an der Hinterwand ein physiologisch schwacher Ort, die sogenannte Laimersche Stelle, die eine Lücke in der Muskulatur darstellt. Genau von dicser Stelle nehmen die Zenkerschen Grenzdivertikel ihren Ausgang, was gewiß kein zufälliges Zusammentreffen ist.

Wenn man dann weiter bedenkt, daß im höheren Alter noch eine Häufung dieser schwächenden Momente eintritt, dic sich hauptsächlich in Atrophie der Muskulatur und des Bindegewebes kennzeichnen, so hat man schließlich auch die Erklärung, warum die Ösophagusdivertikel erst im späteren Alter, meist in den Jahren nach 40 auftreten. Und diese Tatsache möchte ich hauptsächlich gegen die Annahme ins Feld führen, daB nur entwicklungsgeschichtliche Störungen dazu Veranlassung geben.

Auch Spasmen können die Ursache für eine Divertikelbildung abgeben. Bekannt ist, daß der Krampf an der Cardia, der sogenannte Cardiospasmus, zu einer diffusen Erweiterung der ganzen Speiseröhre führt. Weniger bekannt ist aber, daß auch am Beginn der Speiseröhre, dem von Killia n gefundenen "Osophagusnund, cin ähnlicher Krampf rorkommt. Killian, A l b r e cht und andere haben diesen Krampf des Ösophagusmundes wiederholt beobachtet. Die Folge dieses Ösophagospasmus wird ebenfalls cine Ausweitung der Speiseröhre sein. Und daB es dabei nicht zu einer allgemeinen Erweiterung, sondern nur zu einer teilweisen kommt, dafür ist die Erklärung darin zu suchen, daß sich auch hier der Druck vor allem an der schwächsten Stelle, 
nämlich dem Laimerschen Fleck bemerkbar machen vird. Ist einmal eine kleine Aussackung gegeben, dann besorgen die Speisen und der Schluckakt schon das weitere. Die Speisen, die in diese Aussackung geraten, bleiben dort liegen und üben einen dauernden Druck aus. Diesen Druck vergrößert der durch den Schluckakt hervorgerufene Überdruck noch weiterhin. Über die Entstehungsart der Divertikel auf dieser Basis sagt $\mathrm{K}$ illi a folgendes: „Der Ösophagospasmus führt zu einer particllen Erweiterung des Hypopharynx, dem sogenannten Pulsionsdivertikel" . Andere Autoren freilich legen diesem Krampf nicht diese große Bedeutung zu. So glaubt nämlich Ju rasz, daß der Ösophagospasmus nicht das Primäre sei, sondern erst sekundär aufträte infolge des Divertikels, und stützt diese Anschauung mit der Beobachtung, daß nach Beseitigung des Divertikels auch der Ösophagospasmus geschwunden sei.

Ein weiterer Punkt, der für das Auftreten von Ösophagusdivertikeln von Belang sein kann, ist das Jrauma. Es liegt eigentlich auf der Hand, daß nach Verletzungen Aussackungen der Speiseröhre entstehen können. v. Bergmann sagt darüber, nachdem er die Entstehung von Divertikeln auf Grund von Paralysen abgelehnt hatte: ,Mit mehr Wahrscheinlichkeit dürfte man, wie das von Fricdeberg geschehen ist, auf ein Trauma als ursächliches Moment zurückgreifen".

Und daß das Trauma gerade an der kritischen Stelle des Ösophagus einen Angriffspunkt findet, erklärt sich wieder aus dem Bau der Speiseröhre. Denn gerade an dem hinteren Ringknorpel an der ersten physiologischen Enge werden Fremdkörper im Halse am leichstesten stecken bleiben. Es können sich dort alle möglichen Fremdkörper fangen, wie spitze Knochen, Fischgräten, Obstkerne, verschluckte Gebisse, harte Brotkrusten, scharfrandige und spitze Eicrschalen. Auch Verätzungen und Verbrennungen durch heiße Speisen gehören hierher. Die enta zündlichen Prozesse, die sich im Anschluß an solche Traumen einstellen, führen zu ciner Schädigung und Schwächung der Wand und damit zu einer Disposition für die Entstehung von Divertikeln.

Die Durchsicht der Literatur ergibt eine Reihe von Belegen für obige Behauptungen. So berichtet Schlesinger über ein Ösophagusdivertikel an der Hinterwand, das im An- 
schluß an ein Trauma entstanden war. Tus a sah selbst bei einem I. 6 jährigen Mädchen, das drei Jahre vorher eine Eierschale verschluckt hatte, ein Ösophagusdivertikel entstehen und er macht noch Mitteilung über einen gleichen Fall aus der Literatur. Klose und $\mathrm{P}$ a u $\mathrm{l}$ erwähnen als Ursache eine stecken gebliebene Karpfengräte, $K$ ü h n e eine steckengebliebene Brotkruste, $L$ u d l o w einen steckengebliebenen Kirschkern, der nach drei Tagen cntfernt wurde. Der Patient B e rk h a n s' verschluckte mit i I Jahren 2 Hannoversche Dreier. Bei dem Falle v. Hackers begann das Leiden mit Wundgefühl in Halse nach dem Genuß heißer Suppe und Göppert gibt einer Verbrennung mit Rindfleisch die Schuld. Overk a m p weiß über eine eitrige Halsentzündung zu berichten, die 4 Jahre vor Beginn des Leidens aufgetreten war. Bayers Patient hatte als Kind eine eiternde Ilalsentzündung. gehabt und war deswegen operiert worden. Ganz ähnlich ist die Beobachtung E.v. Bergm a n n s: Fine 38 jährige Frau, die in ihrem 8. Lebensjahr an einer Eiterung an Halse gelitten hatte, bekam ein Ösophasgusdivertikel, das schwerste Erscheinungen hervorrief und durch Operation geheilt wurde. J u ras z beschuldigt für die Entstehung des Divertikels das Steckenbleiben eines Knochens im Halse, das 1/2 Jahr vorher erfolgte und sagt. „Es ist naheliegend, das Trauma in ursächlichen Zusammenhang mit der Entstehung des Divertikels zu bringen". Jenkel führt in seinem Falle die Entstehung des Divertikels auf ein vor ro Jahren verschlucktes kleines Knochenstück zurück. Aus der VeterinärMedizin liegt eine interessante Beobachtung von $\mathrm{M}$ a u ri vor, der bei einem Esel ein 3 Ltr. fassendes Ösophagusdivertikel fand, das durch Trauma entstanden war. Durch einen RiB in der Muskulatur hatte sich die Schleimhaut vorgebuchtet und zur Bildung dieses riesigen Divertikels geführt.

Von den traumatischen Ursachen sind dann noch Stenosen zu erwähnen. Wenn auch die Strikturen meist zu einer diffusen Erweiterung der Speiseröhre Anlaß geben, so kann doch auch einmal sich darüber ein Pulsionsdivertikel entwickeln. Sargnon beschreibt einen Fall, wo sich ïber einer impermeablen Striktur cin Divertikel ausgebildet hat. B a ue $\mathrm{r} f \mathrm{feinds}$ Patient hatte eine angeborene Ösophagus-Stenose und im Falle Overkamps hatte einen Tumor am Halse eine Stenose der Speiseröhre ver- 
ursacht und damit zur Bildung eines Divertikels Anlaß gegeben. Flesch berichtet über ein 15 Jahre altes Mädchen, das nach Natron-Lauge-Verätzung eine narbige Stenose der Speiseröhre bekam und wo man bei der Sektion dann über der Stenose ein Divertikel fand. Guise $z$ hat Ioo Präparate von Ösophagusdilatationen und Divertikeln daraufhin untersucht und ist $z u$ dem Ergebnis gekommen, daß die Divertikel und Dilatationen sekundäre Bildungen über Verengerungen der Speiseröhre sind. Es liegen also immerhin genug Handhaben vor, um die oben aufgestellte Behauptung zu stützen. Alle diese Angaben müssen daher unsere Aufmerksamkeit auf das Trauma als Ursache hinlenken. Wenn wir aber nicht noch häufiger eine traumatische Ätiologic festgestellt finden, so liegt dies wohl daran, daß meist danach gar nicht gefragt wird. Und wenn man auch danach forscht, machen die Patienten keine Angaben, weil sie das Trauma in der Regel schon längst wieder vergessen haben.

Die Diagnose des Ösophagusdivertikels kann manchmal sehr leicht sein, manchmal jedoch große Schwierigkeiten bieten und erst nach Anwendung aller klinischen Untersuchungsmethoden gestellt werden. Oft gibt eine genau erhobene Anamnese allein schon den klaren Hinweis und den richtigen Aufschluß über dic Art des vorliegenden Leidens.

In unserem $\mathrm{I}$. Fall muß man die Schilderung der Beschwerden von seiten des Kranken als geradezu klassisch für ein Ösophagusdivertikel ansprechen. Die seit längerer Zeit -5 Jahren - bestehenden Schluckbeschwerden und das Gefühl von Steckenbleiben der Speisen im Halse deuten auf eine Passagebehinderung in der Speiseröhre hin. Freilich können wir noch nicht sagen, welcher Art dieselbe ist.

Die Ursache dafiir könnte ja ebensogut eine carcinomatöse oder narbige Striktur sein. Diese Angaben aber werden uns weiterbringen, wenn wir hören, daß Speisen anfangs ganz gut hinuntergingen, dann aber neue Bissen, ganz unabhängig von ihrer Größe, plötzlich stecken blieben und nicht mehr hinunter gebracht werden können. Bei anderen ist es wieder umgekehrt. Da bleiben gerade die ersten Bissen stecken und die folgenden gehen danı glatt hinunter. Nach Schwarzenbach erklärt sich dies daraus, daß eine kleine Ausbuchtung nur so lange 
die Passage der Bissen behindert, bis sie ganz ausgefüllt ist". In dem anderen Falle findet es seine Erklärung darin, daß der gefüllte Sack des Divertikels eben das Lumen der Speiseröhre drückt und so das weitere Durchgehen von Speisen und Flüssigkeit unmöglich macht. Wenn wir dann erfahren, daß anfangs feste Speisen noch hinunter gehen, jetzt aber schon breiige Nahrung, ja manchmal Flüssigkeit Beschwerden macht, so ergibt sich daraus nur ein Größerwerden des Hindernisses. Und hören wir, daß nach Nahrungsaufnahme mit viel Schleim vermengte Speisen wieder hochkommen, die erst nach öfterem Kauen und mehrmaligem Schlucken und Pressen hinunter zu bringen sind, so wird der Verdacht auf ein Ösophagusdivertikel sehr nahe gelegt. Geradezu herausgefordert wird aber diese Diagnose durch die Angaben des Kranken, er könne oft am besten schlucken, wenn er den Kopf recht weit nach vorn beuge oder nach verschiedenen Seiten hin drehe. Diese Manipulationen sprechen dafür, daB das Hindernis nur bei einer bestimmten Lage in Erscheinung tritt, also nicht in einer narbigen oder carcinomatösen Striktur gelegen sein kann. Daß beim Vorbeugen des Kopfes die Speisen besser durchgehen, ist begreiflich, wenn man bedenkt, daß der Eingang zum Divertikel von der Hinterwand ausgeht. Es können aber , tuch andere Lagen und Stellungen die Passage am leichtesten frei machen. Bei einigen gehen die Speisen besser hindurch, wenn sie am Rücken liegen. N e u k i r h hat bei seinem Patienten greschen, daß der vorher unwegsame Ösophagus in Seitenlage für Speisen und Getränke wieder durchgängig wurde.

Hat uns einmal die Anamnese den Verdacht auf ein Ȯsophagusdivertikel geweckt, dann ist bis zu dessen Feststellung in der Regel kein weiter Weg mehr. Die objektive Untersuchung gibt uns bald dic sicheren und zwingenden Beweise an die Hand. Finden wir nach dem Schlucken von Flüssigkeit oder nach Nahrungsaufnahme eine Anschwellung am Halse, einen Kropf, wie die Patienten sich meist ausdrücken, so ist dies schon ein wichtiger Fingerzeig. Diese Anschwellung wird manchmal so groß, daß man sie mit der Hand ausdrücken kann ( $\mathrm{Sch}$ war $z$ enbach), oder durch streichende Bewegungen wieder zum Verschwinden bringen kann ( $\mathrm{u}$ rasz, Payr). 
Ebenso wirhtig ist eine durch Perkussion nachweisbare Dämpfung, die vorher nicht vorhanden war. Das Auftreten einer solchen Dämpfung weist darauf hin, daß es sich natürlich um einen Sack handeln muB, der sich eben bei der Nahrungsaufnahme gefüllt hat. Mit Sicherheit darf man jedoch auf diese Zeichen nicht rechnen. Von beiden ist das zweite noch am häufigsten. Aber auch dies wird in einem verhältnismäßig geringen Prozentsatz beobachtet. In meinem I. Falle läßt sich in der Tat eine kleine Dämpfung nachweisen, die vor dem Schluckakt nicht bestanden hatte. In dem anderen Falle dagegen fehlt sie.

Eine: Anschwellung am Halse, wie sie von anderer Seite gefunden wurde (Pfister, Jurasz, Payr, Curschinann, Le $x$ e r), konnte ich nicht feststellen. Diese sichtbare Geschwulstbildung wird ja auch nur in ungefähr 39 Proz. der Fälle beobachtet (L o theissen).

Von größerer Bedeutung sind glucksende und gurgelnde Geräusche, die man beim Schlucken hört. Ihr Vorkommen ist aber ebenfalls nicht gar zu häufig. Im Falle Th. fehlt $\epsilon s$, während es auffallenderweise bei dem Pat. M. mit dem kleineren Divertikel vorhanden ist.

Von underen Erscheinungen, die freilich weniger exakt auf ein Ösophagusdiverdikel als vielmehr auf einen Raum beengenden Proze $B$ hinweisen, sind Nervenstörungen zu erwähnen. Am häufigsten kommen die Lähmungen des Nervus recurrens in Betracht, die dann in Heiserkeit ihren Ausdruck finden. Weiter hat man Sympathikusstörungen gefunden. Is a a $\mathrm{k}$ sah einmal das ausgesprochene Bild des Hornerschen Symptomenkomplexes, der sich in Myosis, Enophthalmus und Lidspaltenverengerung kennzeichnet. Auch stärkere Verdrängungen der Gefäße können durch ein sich vergrößerndes Divertikel hervorgerufen werden. Dieselben werden in der Regel einscitig sein.

Beim ausgesprochenen Krankheitsbilde gibt uns also schon die Anamnese, sowie die Untersuchung mittels Perkussion und Auskultation ziemliche Klarheit über die vorliegende Krankheit. Aber nicht jedesmal sind beim Divertikel alle Erscheinungen in so klassischer Form ausgeprägt und wir werden daher zu weiteren klinischen Untersuchungsmethoden unsere Zuflucht nehmen müssen. 
In erster Linie kommt da die Sondicrung in Betracht. Die Sonde war früher das wichtigste Instrument bei der Unter. suchung des Ösophagusdivertikels und erst ihre Anwendung hatte die Klarstellung vieler Fälle ermöglicht. Man benutat dabei entweder die gewöhnliche Sonde oder die sogenannte Divertikelsonde. Um nämlich das Auffinden des Divertikels zu erleichtern, hat man eine Sonde konstruiert, die eine dem Mercierkatheter ähnliche Krümmung aufweist. Je nachdem die Spitze der Krümmung nach vorn oder hinten sieht, gleitet die Sonde am Divertikel vorbei oder fängt sich in demselben.

Alle Berichte über Sondierung bei Pulsionsdivertikel des Osophagus enthalten die Angabe, daß man noch $18-23 \mathrm{~cm}$ hinter der Zahnreihe das Hindernis findet. Meist handelt es sich um einen Abstand von $20-22 \mathrm{~cm}$ (Albrecht). Jch fand es einmal in $22 \mathrm{~cm}$, ein andermal in $2 \mathrm{I} \mathrm{cm}$ Entfernung hinter der Zahnreihe. Es wäre aber grundfalsch, aus einer größeren Distanz den Schluß zu ziehen, in diesem Falle ein Grenzdivertikel auszuschließen. I)er Broden des Dirertikelsackes kann nämlich je nach der Größe bis zum Aortenbogen und noch tiefer :eichen. So sah Schmiegelow im Röntgenbild ein mächtiges Divertikel, dessen unterer Rand auf der Höhe des 5. Brustwirbels stand. Bei der Sektion fand man dann ein typisches Zenker, sches Divertikel. Erstreckt sich nun das Divertikel recht tief in den Thorax hinab, so wird man selbstverständlich bei der Sondierung tiefer kommen, bis man einen Widerstand fühlt. Aber trotzdem haben wir ein Divertikel vor uns, dessen Mund auf der Höhe des Cricoidknorpels liegt, und das demnach zur Gruppe der pharyngo-ösophagealen Divertikel gehört.

Kommt man nach Einführung der Sonde in der cben beschriebenen Entfernung hinter der Zahnreihe auf ein Hindernis und finden wir dieses Hindernis bei wiederholter Untersuchung, so wird unser Verdacht nur bestätigt. Dieser Verdacht verdichtet sich zur vollen Gewißheit, wenn bei einer anderen Untersuchung die Sonde an dem früher gefundenen Hindernis vorbeigleitet und sich glatt in den Magen einführen läßt. v. Bergmann sagt über diese Erscheinungen: „Das Symptom des Steckenbleibens einmal und anstandslosen Hinabgleitens ein 
andermal ist für die Diagnose des Ösophagusdivertikels entscheidend".

Wir können das Ergebnis noch weiter dadurch sichern, wenn es uns glückt, zwei Sonden in der Weise einzuführen, daß die eine in das Divertikel, die andere in die Speiseröhre und den Magen zu liegen kommt. Damit ist durch Sondierung der einwandfreie Beweis für das Vorhandensein eines Divertikels erbracht. Aus alledem ist zu ersehen, da $\beta$ die Diagnose mittels der Sonde wohl möglich, aber doch recht umständlich und nicht gerade angenehm ist.

Einen großen Fortschritt in der Erkennung des Ösophagusdivertikels hat uns das Ösophagoskop gebracht, das uns einen direkten Blick auf den Abgang sowie in den Sack des Divertikels werfen läßt und dessen Aussehen aufdeckt. ,Ein absolut sicherer Beweis für das Bestehen eines Divertikels läßt sich auf ösophagoskopischem Wege nach v. Eicken dann erbringen, wenn es gelingt, unter Kontrolle des Auges aus dem Divertikelsack über die Schwelle in den Ösophagus und umgekehrt, aus dem Ösopha. gus über die Schwelle in das Divertikel zu gelangen." Um sich diese Manipulationen zu erleichtern, ist es jecloch sehr empfehlenswert, bei der Untersuchung einen sogenannten Divertikeltubus $\mathrm{zu}$ verwenden, weil es sonst leicht möglich ist, daß tnan den Eingang des Divertikels nicht findet. Man braucht nicht eigens betonen, daß es eine große Fertigkeit in der Behandlung dieses Instrumentes voraussetzt, wenn man diesem Postulat genügen will. v. Eicken selbst sagt: „Die Untersuchung eines Divera tikelpatienten erfordert Ubung und Gewandtheit in der Handhabung des Ösophagoskops, und gehört zweifellos zu den schwierigeren Aufgaben dieser Methode." Und Fiedler gesteht, nachdem er für die Ösophagoskopie warm eingetreten ist: „Es stimmen sämtliche Untersucher darin überein, daß hier wie bei keiner anderen Methode Übung das erste Erfordernis ist."

Dieses Instrument wird also nur in der Hand erfahrener Spezialisten seine vollen Früchte tragen und damit cntfällt es schon von vornherein für die gewöhnliche Untersuchung.

An seine Stelle tritt ein anderes Verfahren, das uns viel einfacher und bequemer den Weg zur Diagnose des Ösophagusdivertikels weist, nämlich die Röntgenuntersuchung. Die Rönt- 
genstrahlen haben in der Tat die Diagnostik des Pulsionsdivertikels mächtig gefördert. Den Beweis hierfür bringt ein Blick auf die Literatur. Während man in den Jahren vor 1896 nur wenige Veröffentlichungen über Ösophagusdivertikel findet, bemerkt man seit der Entdeckung der Röntgenstrahlen eine auffallende Zunahme der Publikationen. In der ersten Zeit wurden Sonden in das Divertikel eingeführt, ev. auch zwei, wie bei der üblichen Sondenuntersuchung und dann wurde eine Durchleuchtung vorgenommen oder eine Aufnahme gemacht. Ja man hat auch versucht, mit dem Magenschlauch Wismut in den Sack zu bringen, um ihn auf diese Weise zu füllen. Dieses umständliche Verfahren, das natürlich zu Fehldiagnosen Anlaß geben mußte, hat man aber längst verlassen und man läßt jetzt fast nur mehr irgendein Kontrastmittel schlucken, um auf diesem natürlichen Wege das Divertikel zu füllen.

Heutzutage gestaltet sich die Technik der Röntgenuntersuchung sehr einfach. Bei der Durchleuchtung stellt inan den Patienten vor den Schirm, läßt ihn eine Wismut- oder Bariumaufschwemmung trinken und beobachtet nun den Weg dieser Flüssigkeit. Das Beobachtungsergebnis ist ein verschicdenes. Manchmal füllt sich der Sack sofort und es erscheint bei sagittaler Durchleuchtung meist ein größerer oder kleinerer Schatten zwischen Schildknorpel und Jugulum.

Dieser Schatten zeigt halbmondförmige Gestalt mit nach unten scharf konvexer Begrenzungslinie, während der obere Rand meist unscharf ist und in horizontaler Richtung verläuft. Beim Schlucken steigt der Schatten auf und $a b$ und verändert auch manchmal seine Größe.

Ein andermal kann man beobachten, daß anfangs die Wismutaufschwemmung glatt hinunter geht; erst allmählich bildet sich dann der oben beschriebene Schatten, der bei weiterem Schlucken von Wismut an Intensität und Größe zunimmt. Nach der Durchleuchtung in sagittaler Richtung empfiehlt sich eine Durchleuchtung in einem schrägen Durchmesser. Der Kranke wird dazu meist in die sogenannte Fechterstellung gebracht und man kann nun recht gut die Ausdehnung des Divertikels nach vorn und hinten zur Anschauung bringen. Nach Füllung des Divertikels kann man öfters beobachten, daß beim Schluck. 
akt das Divertikel gewissermaßen überläuft und das Kontrast. mittel dann in die Speiseröhre fließt. Dabei verkleinert sich der Divertikelsack, sei es nun, daß ein Druck von der Umgebung her dazu führt, sei es, daß eine aktive Muskcolkontralition dies bedingt.

Nach der Durchleuchtung wird man zur Fixierung des Befundes eine Röntgenaufnahme vornehmen. Man macht àm besten auch hier zwei Aufnahmen, eine bei ventrodorsalem Strahlengang, eine zweite in schräger Richtung. Letztere wird am besten so vorgenommen, daB man den Patienten in linke Schräglage bringt. Er liegt dann mit der linken Seite der Platte an und die Röntgennöhre wird rechts vor den Brustkorb postiert. Die .Ourchleuchtung sowie die Aufnahme werden im Stehen vorgenommen. Unter Umständen kann aber auch eine Aufnahme im Liegen wünschenswert sein, nämlich dann, wenn es sich darum handelt, das Divertikel in sciner ganzen Ausdehnung zur Darstellung zu bringen.

$\Lambda$ uf diese Weise gewinnt man Röntgenbilder, die cinen so klaren, eindeutigen und anschaulichen Befund geben, svie man ilu sich besser nicht wünschen kann. Und Goldam me $r$ hat daher vollkommen recht, wenn er sagt : „Auf den Röntgenogramm läßt sich die Diagnose wie von einem Buche ablesen."

Ich möchte nun auf die Röntgenuntersuchung meines ersten Falles noch näher eingehen, da sie neben dem Aufzeigen des Divertikels noch in doppelter Hinsicht Interessantes bietet. Es ist, soweit mir bekannt, zum ersten Male gelungen, das Divertikel in seiner ganzen Größe und Ausdehnung auf dem Röntgenogramm darzustellen. Ka u $\mathrm{m}$ an $\mathrm{n}$ und Kienboeck schreiben nämlich: „In allen unseren Fällen zeigt sich, daß es nicht mög. lich war, das Divertikel mit Wismutbrei in toto zu füllen, so dab es in seiner ganzen Größe und Form und im Zusammen. hang mit dem Pharynx zu sehen wäre." Auf meinem Röntgeno* gramm ist nun in schönster Weise zu sehen, wie das Divertikel vollkommen mit Brei gefüllt ist. Es hat zwerchsackähnliche Form. 1)ie obere Hälfte sendet einen nach oben ausgehenden Zapfen aus und darüber befindet sich damn ein weiterer Schatten, der nach oben hin unscharfe Begrenzung zeigt. Es kann wolıl kein $Z$ weifel bestehen, daß der nach oben gehende Fortsatz den 
Mund des Divertikels darstellt und daß es sich bei dem darüberliegenden Schatten um eine teilweise Füllung des Pharynx handelt. Diese Divertikeldarstellung glaube ich in erster Linie dem Umstand zuschreiben zu müssen, daß die Aufnahme im Liegen gemacht wurde, wodurch eben die Füllung des Divertikels in seinem Längsdurchmesser möglich wurde. Ich möchte daher zu diesem Zwecke die Aufnahme im Liegen noch besonders befürworten.

Dieser Fall hat ferner wegen einer 2. Beobachtung noch eine gewisse Bedeutung. Wir bekommen nämlich genauen AufschluB über die Verweildauer des Breies im Divertikel. Am Tage nach der Untersuchung ist das Divertikel noch zur Hälfte mit Brei gefüllt. Nach 5 Tagen findet man noch einen kleinen Rest, der genau so aussieht, wie der Rückstand bei schwerer Motilitätsstörung des Magens. Erst nach 6 Tagen erweist sich das Divertikel als leer. Im Falle $M$. enthält das Divertikel am nächsten Tage noch etwas Brei. Ain 3. Tage ist jedoch kein Brei mehr nachzuweisen. Daraus ergibt sich, da $B$ sich die kleineren Divertikel rascher entleeren wie die großen, was ja nach dem ganzen Befunde nicht anders zu erwarten ist.

Eine ähnliche Beobachtung hat bis jetzt nur Becker gemacht, der noch nach 8 Tagen Wismutbrei im Divertikel vorfand. Rosenthal bcobachtete bei seinem Kranken, daB er einmal Rosinen regurgitierte, die er vor 8 Tagen gegessen hatte (nach $\mathrm{Starck}$ ). Aus der vorröntgenologischen Zeit liegt ein diesbezüglicher Bericht von Ayres vor. Eine 38 jährige Frau mit Ösophagusdivertikel warf Teile einer Speise aus, die sie nachweislich vor 6 Wochen gegessen hatte. Diese Beobachtung stellt somit das längste Verweilen von Speise im Divertikel dar. Meiner Ansicht nach sind diese Befunde gewiB keine zufälligen. Längeres Verweilen des Breies im Divertikel gehört wohl sicher zur Regel. Der Grund, warum wir dies jedoch so wenig beschrieben finden, dürfte darin $z u$ suchen sein, daß daraufhin nicht regelmäßig untersucht wurde, weil man sich meist mit der Feststellung des Divertikels schon begnügte. Sonst hätte man diese Beobachtung entschieden häufiger machen müssen. Die Röntgenstrahlen geben uns also nicht nur Klarheit über das Vorliegen eines Divertikels, sondern verschaffen uns 
auch Aufschluß über dessen Form und Größe und die Verweildauer des Breies im Divertikelsack.

Wägen wir Vor- und Nachteile der einzelnen Methoden gegeneinander $a b$, so werden wir unbedingt dem Röntgenverfahren den Vorzug geben müssen.

Die Sonde hat wohl den Vorteil, daß man sie jederzeit rasch zur Hand hat und daß sie keine besondere Technik erfordert. Andererseits ist aber die Einführung doch manchmal recht unangenehm, ja nicht vollkommen ungefährlich. Ist es doch bei der gewöhnlichen Sondierung vorgekommen, daß die Divertikelwand durchstoßen und hierdurch der Tod des Patienten heraufbeschworen wurde (v. Bruns, König, Völker, Müller). Jurasz will deshalb die Sondierung von vornherein vermieden wissen. Auch ist noch zu erwähnen, daß man manchmal das Divertikel mit der Sonde nicht wird feststellen können, weil es eben nicht gelingt, die Sonde in den Sack zu bringen. Die Sondierung stellt übrigens nur das Vorhandensein eines Divertikels fest, über Größe und Form sowie über das Divertikelinnere sagt sie nichts.

Was nun die Ösophagoskopie betrifft, so muß sie wegen der Schwierigkeit ihrer Teclnnik von vornherein gewandten Spezialisten überlassen bleiben. In diesen Händen wird sie freilich recht Vortreffliches leisten. Sie zeigt den Abgang des Divertikelmundes genau an, informiert über ev. vorhandenen Inhalt, vor allem aber über das Aussehen der Schleimhaut. Als Nachteil fällt aber sehr ins Gewicht, daß sich nicht alle Leute zur Ósophagoskopie eignen. Nach statistischen Erhebungen ist die Ausführung der Ösophagoskopie überhaupt in $1-2$ Proz. nicht möglich. Ja Kirstein spricht einem Viertel der erwachsenen Menschen die anatomische Disposition zur Ösophagoskopie ab (Fiedler).

Meist handelt es sich dabei um alte Leute, deren Wirbelsäule so starr ist, daß die Einführung des Ösophagoskops unmöglich wird. Auch weit vorspringender Oberkiefer kann ein Hindernis abgeben. Endlich gibi noch starke Nervosität eine Kontraindikation. Und wenn die Ösophagoskopie anch gelingt, so ist damit noch nicht alles gewonnen. Starke Schleim- und Speichelsekretion, pulsatorische und reflektorische Bewegungen der Wandung, reflektorische Würgbewegungen und Erbrechen können das Er- 
gebnis sehr beeinträchtigen. Auch die Deutung der Bilder ist nicht leicht und erfordert nach Starck „eine große Übung: an einem reichlichen Material". Man kommt deshalb of mit einer Untersuchung nicht aus, sondern muB manchmal 2-3 mal die suspekte Stelle aufsuchen. Was dies bei alten Leuten zu bedeuten hat, die sich überhaupt recht ungern zu solchen Untersuchungen herbeilassen, weiß jeder.

Dann ist es immerhin keine Kleinigkeit, die von v. E i cke n aufgestellten Forderungen für die Diagnose eines Ösophagusdivertikels $\mathrm{zu}$ erfüllen. Dies beweist schon die Tatsache, daB es einem Meister in der Ösophagoskopie wie Killian nicht immer glückte, das Ösophagoskop in das Divertikel und dann in die Speiseröhre einzuführen, oder umgekehrt, um damit obigen Forderungen gerecht zu werden. Auch S ch mil in sk y berichtet, daß er den Eingang in den Ösophagus öfters nicht finden konnte. Um wieviel häufiger wird ein weniger geübter Untersucher, ein Fiasko erleben. Nach v. E i ck e n kommt nämlich dieser Mißerfolg nicht so selten vor, wenn eine Entzündung oder Stenosierung des Ösophagus vorliegt. Es gelingt dann nicht die Schwelle des Divertikels oder den Eingang zum Ösophagus zu finden. Und glücken alle Manipulationen, so ist doch nicht zu leugnen, daß die Ösophagoskopie mit recht viel Unannehmlichkeiten verbunden ist.

Schon die Lagerung und Einführung des Ösophagoskops selbst, mag sie nun im Sitzen oder in Rückenlage vorgenommen werden, bringt recht lästige Beschwerden. Diese werden noch gesteigert bei den Manövern, die die Aufsuchung des Divertikelmundes bezwecken. Um eine ruhige Untersuchung zu ermöglichen, müssen wir in der Regel zur Anästhesierung unsere Zuflucht nehmen, sei es, daß man zu einer Allgemeinnarkose greift, sei es, daß man von der Cocainisierung der Schleimhaut Gebrauch macht. Und endlich ist nicht $\mathrm{zu}$ vergessen, daß die Ösophagoskopie noch mehr wie die Sondierung Gefahren in sich schließt. Es sind dies Perforation des Divertikels und Ruptur eines etwa bestehenden Aortenaneurysmas. So sind 3 Patienten Gott. steins an Perforation gestorben und Jackson berechnet I Proz. Todesfälle für die ösophagoskopische Untersuchung. Es wird daher die Forderung erhoben bei der Untersuchung auf 
Ósophagusdivertikel die Ǒsophagoskopie überhaupt erst zuletzt anzuwenden, ja sie zu unterlassen, wenn die Möglichkeit eines vorhandenen Aorlenaneurysmas gegeben ist ( $M$ a y e $r$ ).

Für die Röntgenuntersuchung fallen alle diese Mißhelligkeiten weg. Die lagerung ist weder im Sitzen noch im Liegen unangenehm, Anästhesierung kommt von vornherein nicht in Betracht. Beschwerden und Gefahren sind daher bei dieser Untersuchung nicht gegeben. Als ev. Nachteil möchte ich die Frage der Versager erörtern. Soweit ich die Literatur überblicke, kann ich keinen Fall finden, wo die Röntgenuntersuchung bei der Diagnosestellung des Ösophagusdiverdikels im Stiche gelassen hätte. Der von $\mathrm{Barrow}$ und $\mathrm{Cunning}$ berichtete Mißerfolg zählt nicht, weil dabei ein viel zu kompliziertes Verfahren angewendet wurde. Es wurde nämlich versucht, mittels des Magenschlauchs das Divertikel mit Wismut zu füllen, statt einfach eine Wismut- oder Bariumaufschwemmung schlucken $\mathrm{zu}$ lassen.

Auch im Falle K üsters, wo die Diagnose auf Divertikel gestellt wurde, während es sich um eine Dilatation über einer Narbenstriktur handelte, trifft das Röntgenbild keine Schuld. K üster sagt ja selbst, daß eine genaue Analyse des Röntgenogramms, das einen deutlichen Fortsatz am unterem Rande des Schatt'ens zeigte, den Irrtum hätte vermeiden lassen und den Weg zur richtigen Diagnose hätte zeigen müssen.

Und so bleibt nur noch der von $K r$ a use veröffentlichto Fall zu besprechen, bei dem das Röntgenbild eine FehIdiagnose veranlaßt hatte. Es handelte sich um einen 52 jährigen Mann, der seit 2 Jahren über Schluckbeschwerden klagte und bei dem sich eine ausdrückbare Geschwulst am Halse gebildet hatte. Das Röntgenbild zeigte einen halbmondförmigen Schatten über dem Herzschatten, und zwar war dieser Befund bei wiederholter Untersuchung vorhanden. Röntgen- und Sondenuntersuchung fülrten daher im Verein mit der Anamnese und dem klinischen Befund zur Diagnose Divertikel. Bei der Operation aber fand man, daß kein Divertikel, sondern ein Carcinom vorlag. Die Sektion klärte dann die Verhältnisse auf. Ein hochsitzendes Osophaguscarcinom war perforiert und um die Perforalionsstelle hatte sich ein beträchtlicher, bindegewebiger Sack gebildet, der 
sich mit Speisen füllte. $\mathrm{DaB}$ hier das Röntgenbild zur Diagnose Divertikel Anlaß gab, wundert nicht. Waren doch klinisch wie röntgenologisch alle Merkmale eines solchen vorhanden, wie Schluckbeschwerden, ausdrückbare Gescliwulst am Halse, positiver Sondenbefund und Röntgenbild mit nach inten halbmondförmiger scharfer Begrenzung. Und schlieblich lag auch, wenn auch nicht dem Namen, so doch dem Wesen nach eine Art Divertikel vor. Wie es nämlich sonst durch Ausweitung einer schwachen Stelle der Speiseröhre zu einer Sackbildung kommt, so führte hier der Durchbruch eines Ösophaguscarcinoms zur Entstehung eines neben der Speiseröhre gelegenen und mit ihr in Verbindung stehenden bindegewebigen Sackes, der sich genau so wie ein Divertikel mit einem Teil der Speisen füllte. Zum Bilde des wirklichen Divertikels fehlte ja nur die Auskleidung des Sackes mit Schleimhaut. Dieser Fall hat also mit einem Ösophagusdivertikel so viel gemeinsam, daB man nurröntgenologisch ihn nichı davon trennen kann und er fällt damit bereits jenseits der Grenzen dieser Methode. Man kann also hier dem Röntgenverfahren keinen Vorwurf machen.

Andererseits gebe ich $z \mathrm{u}$ bedenken, daB ein Krankheits. bild, wie das eben beschriebene, ein so außergewöhnlich seltenes Ereignis ist, daß man mit ihm eigentlich nicht zu rechnen braucht.

In solch komplizicrten Fällen tritt aber dann das Ösophagoskop in seine vollen Rechte, mit dessen Hilfe man eine sichere Diagnose stellen kann. Wenn ich hier die Vorteile der Röntgenuntersuchung ins Licht gerückt habe, so möchte ich andererseits der Ösophagoskopie nicht zu nahe treten; denn über einige Fragen beim Ösophagusdivertikel wird uns das Röntgenbild die Antwort schuldig bleiben. Aber gerade über diese Fragen, die unter Umständen von der größten Wichtigkeit sein können, wird uns das Ösophagoskop genaue Auskunft geben. Handelt es sich nämlich darum zu entscheiden, ob bei einem Ösophagusdivertikel ein ulceröser oder bösartiger Prozeß mitspielt, ob sich vielleicht auf der Schwelle oder im Sack des Divertikels ein Carcinom entwickelt oder ausgebildet hat, so sagt uns darüber das Röntgenbild nichts.

In dieses Dunkel wird einzig und allein das Ósophagoskop Licht werfen. Es wird den ulcerösen oder carcinomatösen ProzeB 
klipp und klar aufzeigen und damit den wahren Charakter des vorliegenden Leidens erkennen lassen. Alle anderen Methoden können da gegen die Ösophagoskopie nicht aufkommen, denn auf diesem Gebiete ist sie unbestrittene Herrscherin und Meisterin.

Aber trotzdem können wir ruhig behaupten, daß die Röntgenuntersuchung für die Diagnose des Ösophagusdivertikels zur wichtigsten Methode geworden ist. Sie gibt uns in bequemster, einfachster Weise klaren und einwandfreien Aufschluß uiber das Bestehen eines Pulsionsdivertikels, über seine Lage, Größe und Form, sowie über die Verweildauer des Inhalts. Sie hat keine Schmerzen und Gefahren im Gefolge und läßt mit diesen Vorteilen alle übrigen klinischen Untersuchungsmethoden weit hinter sich. Und gerade im Hinblick auf das Zenkersche Pulsionsdivertikel der Speiseröhre pflichte ich $\mathrm{Faulh}$ aber vollkommen bei, wenn er sagt: „Die Unterlassung der Röntgenuntersuchung ist eine größere Sünde, als die Nichtanwendung der Ösophagoskopie."

\section{Literaturverzeichnis.}

I. A l bre cht, Uber das pharyngo-ösophageale Pulsionsdivertikel und seine Operation nach der Goldmannschen Methode. Deutsche med. Wochenschrift I9I4, Nr. 22.

2. Ayres, Ein Fall von prolongierter Retention von Nahrung in einem Speiseröhren-Divertikel. Zentralbl. f. Lar. u. Rhinol. 1908.

3. B axrow-Cunning. Lancet 1905, S. 928.

4. B ergman n, E. v., Uber Usophagusdivertikel. Münchn. med. Wochenschrift $1890, \mathrm{Nr} .46$.

5. - Uber Ósophagusdivertikel und seine Behandlung. Arch. f. klin. Chir. Bd. 43 .

6. Berkhan, Hochgradiges Divertikel des Ösophagus mit Ausgang in Genesung. Berl. klin. Wochenschr. 1889, Nr. II.

7. B $\perp$ um, Ein Beitrag zur Diagnose des Osophagusdivertikels. Zentralbl. f. Lar. u. Rhinol. 1901, S. 158.

8. Edgren, Osophagusdivertikel mit krebsiger Degeneration. Zentralbl. f. Lar. u. Rhinol. I89r, S. 638 .

9. Ehrlich, Zur Kasuistik des Ösophagus-Pulsions-Divertikels. Arch. f. Verdauungskrankheiten, Bd. 18, H. 3.

10. Eicken, v., Die klinische Verwertung der direkten Untersuchungsmethoden der Luftwege und der oberen Speisewege. Arch. f. Lar. u. Rhinol. 1905, Bd. 15. 
II. F a ulhaber, Die Röntgendiagnostik der Speiseröhre-Erkrankungen. Halle I 917 .

12. Fiedler, Uber die Untersuchungsmethoden, welche die Diagnose des Osophagusdivertikels ermöglichen. I.-D. Leipzig 1906.

13. Flesch, Divertikelbildung oberhalb der narbigen Osophagusstenose. Orvosi Hetilap 1913. Zit. nach Zentralbl. f. Lar. u. Rhinol.. I913, S. 512.

I4. Fusa, Ein Fall von Osophagusdivertikel. Zentralbl. f. Lar. u. Rhinol. 1892, S. 497.

I5. Guisez, Uber Pathologie und Ätiologie der sogenannten Divertikel und Dilatationen des Ösophagus. Zit. nach Zentralbl. f. Lar. u. Rhinol. rgi 2.

16. H u b e r, Über das Ösophagusdivertikel. Deutsches Arch. f. klin. Med. Bd. 52 .

17. Is a c, Ein Beitrag zur Kasuistik und Symptomatologie des Osophagusdivertikels. Med. Klinik. I9o9, Nr. I3.

18. J akobs, Ein Pulsionsdivertikel der Speiseröhre. Deutsche med. Wochenschr, I912, Nr. 2 I.

19. Jenkel, Zwei Fälle von Pulsionsdivertikel der Speiseröhre. Münchn. med. Wochenschr. I9I4, Nr. 23, S. 1310.

20. J u rasz, Über Ösophagusdivertikel. Beitr. z. klin. Chir., Bd. 7I, S. 609 .

2r. Ka ufmann u. Ki enboeck, Über Erkrankungen der Speiseröhre. Wien. klin. Wochenschr. rgog.

22. Killian, Zur Geschichte der Ösophagoskopie und Gastroskopie. Deutsche Zeitschr. f. Chir., Bd. 58.

23. - Die direkten Methoden der Ösophagusuntersuchung in den Jahren I 9 I und igi2. Sammelreferat. Zeitschr. f. Lar. u. Rhinol. 1913, S. 445

24. - Die ösophagoskopische Diagnose des Pulsionsdivertikels der Speiserühre. Münchn. med. Wochenschr. I900, Nr. 41 .

25. Krause, über einen interessanten Röntgenbefund bei Ösophaguscarcinom, welcher ein Ösophagusdivertikel vortäuschte. Zeitschr. f. Elektrologie u. Röntgenkunde, Bd. I I, H. Io.

26. K üster, Über Divertikel und zirkuläre Narben der Speiseröhre. Arch. f. klin. Chir. 1907, Bd. 83 .

27. L a $u b$, Zenkersches Pulsionsdivertikel. Wiener klin. Wochenschr. Igor, Nr. II.

28. L o the is sen. Handbuch der prakt. Chir. r913. Bd. 2.

29. M a r u y ma, Beitrag zur Kenntnis des Pulsionsdivertikels der Speiseröhre. Mitteil. aus d. Grenzgeb. 1914, Bd. 28, S. I I3.

30. M a u ri, Ein Fall von Osophagusdivertikel. Zentralbl. f. Lar. u. Rhinol. 1891, S. 579 .

31. N eukirch, Pulsionsdivertikel des Schlundes. Deutsches Arch. f. klin. Med. I 885. Bd. 36 .

32. Pay r, Pulsionsdivertikel der Speiseröhre. Münchn. med. Wochenschr. 1917, S. 1274

33. Rösler, Zur Diagnostik des hochsitzenden Pulsionsdivertikels mittels des Röntgenverfahrens. Fortschritte d. Röntgenstrahlen, Bd. 15, S. 218. 
34. Sargnon, Einige Fälle von Ösophagoskopie, ihre Anwendung in der Diagnostik und Behandlung der Narbenstenose. Zeitschr. f. Lar. und Rhinol. 1907, S. 386.

35. $\mathrm{S}$ jo e gre $\mathrm{n}$, Beiträge zur Kenntnis von Divertikeln in der Speiseröhre. Fortschr. a. d. Gebiet d. Röntgenstrahlen, Bd. I4, S. 1 I7.

36. S chles inger, Ósophagusdivertikel. Wiener klin. Wochenschr. 1903, Nr. 18.

37. Schmiegelow, Fall von Diverticulum oesophagei. Zentralbl. f. Lar. u. Rhinol. 19ro, S. 94 .

38. S chmilinsky, Zwei Fälle von pharyngo-ösophagealem Pulsionsdivertikel. Deutsche med. Wochenschr. I904, Nr. 32.

39. Schwarzenbach, Zur operativen Behandlung und Ätiologie des Osophagusdivertikels. Wiener klin. Wochenschr. I9I3.

40. S t a r ck, Die Divertikel der Speiseröhre. Leipzig I900.

4I. - U Uber eine seltene Form von Ösophagusdivertikel mit Demonstration. Münchn. med. Wochenschr. IģoI, Nr. 23 u. 24.

42. Zenker u. Ziemsen, v. Handbuch der spez. Path, Bd. 7. 